\title{
Caring for patients with cancer in the COVID-19 era
}

\author{
Joris van de Haar ${ }^{1,2,3,18}$, Louisa R. Hoes ${ }^{1,3,18}$, Charlotte E. Coles ${ }^{4}$, Kenneth Seamon ${ }^{4}$, Stefan Fröhling ${ }^{5,6}$, \\ Dirk Jäger ${ }^{7}$, Franco Valenza ${ }^{8,9}$, Filippo de Braud ${ }^{8,9}$, Luigi De Petris ${ }^{10,11}$, Jonas Bergh ${ }^{10,11}$, Ingemar Ernberg ${ }^{12}$, \\ Benjamin Besse ${ }^{13}$, Fabrice Barlesi ${ }^{13,14}$, Elena Garralda ${ }^{15}$, Alejandro Piris-Giménez ${ }^{15}$, Michael Baumann ${ }^{5,16}$, \\ Giovanni Apolone ${ }^{9}$, Jean Charles Soria ${ }^{13}$, Josep Tabernero ${ }^{15}{ }^{15}$, Carlos Caldas ${ }^{4}{ }^{4,17 凶}$ and \\ Emile E. Voest $\mathbb{1}^{1,3 \otimes}$
}

\begin{abstract}
The current COVID-19 pandemic challenges oncologists to profoundly re-organize oncological care in order to dramatically reduce hospital visits and admissions and therapy-induced immune-related complications without compromising cancer outcomes. Since COVID-19 is a novel disease, guidance by scientific evidence is often unavailable, and impactful decisions are inevitably made on the basis of expert opinions. Here we report how the seven comprehensive cancer centers of Cancer Core Europe have organized their healthcare systems at an unprecedented scale and pace to make their operations 'pandemic proof'. We identify and discuss many commonalities, but also important local differences, and pinpoint critical research priorities to enable evidence-based remodeling of cancer care during the COVID-19 pandemic. Also, we discuss how the current situation offers a unique window of opportunity for assessing the effects of de-escalating anticancer regimens, which may fast-forward the development of more-refined and less-toxic treatments. By sharing our joint experiences, we offer a roadmap for proceeding and aim to mobilize the global research community to generate the data that are critically needed to offer the best possible care to patients.
\end{abstract}

T he current COVID-19 pandemic is taking healthcare hostage. With huge numbers of infected patients globally and rapidly increasing numbers of newly diagnosed patients, COVID-19 poses an unprecedented challenge to healthcare systems ${ }^{1}$. However, it is also important to realize that mortality from other diseases, such as - but not limited to - cancer, remains as substantial. Due to the COVID-19 crisis, healthcare professionals face the challenge of profoundly re-organizing healthcare systems at an unprecedented pace, not only to effectively handle the COVID-19 pandemic but also to do so without losing sight of other patient care. So, how can healthcare providers make these very difficult choices and prioritizations in an ethically sound way?

Since 2014, the Netherlands Cancer Institute, Karolinska Institute, Institute Gustave Roussy, Cambridge Cancer Center, Istituto Nazionale dei Tumori di Milano, German Cancer Research Center and Vall d'Hebron Institute of Oncology have been working closely together in a legal entity called 'Cancer Core Europe' (CCE) in order to maximize coherence and critical mass in cancer research $^{2-4}$. Together, the consortium represents $\sim 60,000$ patients with newly diagnosed cancer, delivers $\sim 300,000$ treatment courses and sees $\sim 1,200,000$ consultations annually. Furthermore, $>1,500$ clinical trials are being conducted at $\mathrm{CCE}^{4}$. Since these seven comprehensive cancer centers play important roles both nationally and internationally, CCE decided on 24 March 2020 to collect, translate and compare all guidelines put in place to combat cancer during the COVID-19 pandemic. We focused on adult cancers, as a complicated course of COVID-19 in children is infrequent, which suggests that pediatric cancers need a distinct approach. Some centers act on a case-by-case basis or have adopted general, hospital-wide measures, while other centers have developed detailed guidelines specific for each cancer type. Inevitably, given the paucity of data at this point of the COVID-19 pandemic, most measures adopted have been based on educated assumptions and expert opinions, influenced or supported by information extrapolated from other infectious diseases. As oncologists are normally accustomed to make very careful decisions informed by data from large clinical trials and thoughtful discussions, a reality in which one needs to decide almost immediately what is of more or less importance may feel like practicing medicine in a parallel universe.

In this Perspective, we describe how the seven comprehensive cancer centers in CCE have taken steps to preserve high standards of care for patients with cancer while battling shortages in personal protective equipment, beds, personnel and more. We have provided an overview of these experiences and the many commonalities in general measures and goals (summarized in Table 1). However, it is critical to note that we have also observed differences in specific implementation strategies, which are in part the result of the way healthcare is organized in different countries or the sense of urgency

\footnotetext{
'Division of Molecular Oncology \& Immunology, The Netherlands Cancer Institute, Amsterdam, the Netherlands. ${ }^{2}$ Division of Molecular Carcinogenesis, The Netherlands Cancer Institute, Amsterdam, the Netherlands. ${ }^{3}$ Oncode Institute, The Netherlands Cancer Institute, Amsterdam, the Netherlands. ${ }^{4}$ Cancer Research UK Cambridge Cancer Center, Cambridge, UK. ${ }^{5}$ German Cancer Consortium, Heidelberg, Germany. ${ }^{6}$ Division of Translational Medical Oncology, National Center for Tumor Diseases Heidelberg and German Cancer Research Center, Heidelberg, Germany. ${ }^{7}$ Department of Medical Oncology, National Center for Tumor Diseases Heidelberg and Heidelberg University Hospital, Heidelberg, Germany. ${ }^{8}$ Università Statale di Milano, Milan, Italy. ${ }^{9}$ Fondazione IRCCS Istituto Nazionale dei Tumori di Milano, Milan, Italy. ${ }^{10}$ Department of Oncology and Pathology, Karolinska Institutet, Stockholm, Sweden. ${ }^{11}$ Theme Cancer, Karolinska University Hospital, Stockholm, Sweden. ${ }^{12}$ Department of Microbiology, Tumor and Cell Biology, Karolinska Institute, Stockholm, Sweden. ${ }^{13}$ Gustave Roussy Cancer Campus, Villejuif, France. ${ }^{14}$ Aix Marseille University, CNRS, INSERM, CRCM, Marseille, France. ${ }^{15}$ Department of Medical Oncology, Vall d'Hebron University Hospital, Vall d'Hebron Institute of Oncology, Barcelona, Spain. ${ }^{16}$ German Cancer Research Center, Heidelberg, Germany. ${ }^{17}$ Department of Oncology and Cancer Research UK Cambridge Institute, Li Ka Shing Center, University of Cambridge, Cambridge, UK.

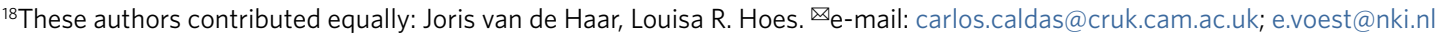


for action in those countries over time, as the COVID-19 pandemic unfolds. Importantly, the absence of guidance from scientific literature, by nature, leads to less-consistent practices, which is reflected across the seven centers. Now and in the time to come, the course of the COVID-19 pandemic will determine how and to what extent oncological care needs to be re-organized. This will probably be a dynamic and quickly evolving process, for which we hope our collective experiences so far can offer guidance to others globally.

\section{Restructuring cancer care during the pandemic}

There are multiple dimensions that the leadership of institutions must consider to be able to create continuity in cancer care during a pandemic: clinical activities, qualified personnel, capacity of care facilities, research activities, and regional and/or national collaborations to collectively share the burden of care for patients with cancer.

Clinical activities. In institutions with dedicated cancer centers (German Cancer Research Center, Institute Gustave Roussy, Istituto Nazionale dei Tumori di Milano and Netherlands Cancer Institute), the general policy has been to attempt to stay COVID-19 free, to ensure that enough clinical and intensive-care capacity could be reserved for critical cancer surgeries or management of side effects of systemic anticancer treatment. This would allow local general hospitals outside of CCE to transfer patients with cancer to the CCE centers for treatment, so the general hospitals could focus on their efforts on treating patients with COVID-19, in addition to those with other diseases requiring urgent care. Therefore, the CCE centers clearly depend on collaborations with local general hospitals and their capacity in their attempts to remain COVID-19 free. Other CCE centers that are located within general hospitals (Cambridge Cancer Center, Vall d'Hebron Institute of Oncology and Karolinska Institute) have had to deal with admissions for patients without cancer that have included suspected and positive cases of COVID-19. Therefore, keeping these centers COVID-19 free was never a realistic or pursued goal.

Given the high transmissibility rate of SARS-Cov-2 (the causative coronavirus for COVID-19), it is the responsibility of all healthcare professionals to ensure patients are not exposed to COVID-19. For CCE centers, this means that face-to-face consultations are now, whenever possible, taking place via web consulting or by telephone calls. For patients with non-urgent appointments that would require them to be physically present in the hospital for routine and follow-up visits or surgeries, those would be postponed as often as possible. In addition, the centers often do not allow visitors to accompany their loved ones when admitted to the hospital or when they go for infusions or radiation treatment, as visitors could potentially be (unknowingly) COVID-19 positive. Patients with symptoms consistent with COVID-19 (but who do not require immediate assessment) are told not to come for their appointments and to follow national guidance on isolation and/or quarantine. As of 2 April 2020, the overall number of patients with cancer admitted to most CCE centers has been $70-80 \%$ of the normal influx.

Adaptation of standard-of-care treatment regimens. Across all centers, standard-of-care treatment regimens have been adapted for two main reasons: (1) to minimize the number of hospital visits and hospitalizations, and (2) to prevent anticancer treatment-induced complications of COVID-19.

In order to minimize hospital visits and hospitalizations, many centers have adopted similar strategies and have issued guidance to convert intravenous treatments to oral or subcutaneous regimens where possible (e.g., hormonal, targeted agents), to switch cytotoxic chemotherapy to less-toxic (monotherapy) treatments to limit the risk of complications requiring hospital admission, or to pause therapies once stable disease (or better) has been established. The current situation has also led to the canceling of non-emergency surgery, including oncological surgery, or replacement of such surgery by radiotherapy. This makes it particularly important to effectively increase radiotherapy capacity, without compromising outcomes (e.g., for breast cancer, which accounts for 30\% of delivered radiotherapy treatment sessions ${ }^{5}$ ).

To prevent anticancer treatment-induced complications of COVID-19, most centers have operated within the paradigm that for anticancer treatments, the added benefit for tumor control should be weighed against the potential risk for COVID-19-related morbidity and mortality. Importantly, although a cancer treatment's added benefit for tumor control may or may not be known from experience and the medical literature, at present there are no robust data with which to quantify treatment-associated risks for COVID-19-related morbidity and mortality. For example, to prevent or shorten neutropenia and lymphopenia, all centers have suggested de-escalating cytotoxic chemotherapy or targeted treatment strategies, or refrain from later (second or subsequent) lines of palliative treatments with upfront low response rates. However, as of 2 April 2020, there is no evidence to support if, and to what extent, neutropenia and lymphopenia are predictive of a negative outcome in the course of COVID-19. Bacterial infections and viral reactivations are well-known complications of neutropenia and lymphopenia, but it is less clear to what extent the severity of de novo viral infections is also affected. Although there are data suggesting that chemotherapy-treated patients with cancer are at risk for severe complications of influenza ${ }^{6,7}$, it is unclear how this might generalize to COVID-19. A recent study has reported increased incidence and morbidity of COVID-19 in patients with cancer ${ }^{8}$, but those findings were rightly challenged by others ${ }^{9,10}$. Anecdotal data have shown a relatively high mortality of 8 of 28 (29\%) hospitalized patients with COVID-19 who were previously diagnosed with cancer ${ }^{11}$. However, the patient numbers were very small, especially for those actively receiving anticancer treatment within the previous 14 days (chemotherapy alone, 2 patients; targeted therapy, 2 patients; immunotherapy and chemotherapy, 1 patient; radiotherapy, 1 patient). Also, it remains unclear to which extent the observed high mortality was driven by anticancer therapy per se, rather than by other clinical characteristics, such as sex, age, frailty and co-morbidities.

As for immune-checkpoint blockade, some CCE centers have issued guidance to deviate from established treatment protocols for safety reasons. For example, (temporary) treatment discontinuation is sometimes advised for patients with stage IV melanoma who have a major and ongoing response while being treated with blockade of the immune-checkpoint receptor PD-1. For some centers, the rationale is based on the observation that patients with COVID-19 seem to have an inflammatory response driven by myeloid cells and lymphopenia, whereas blockade of PD-1 may bias the immune system toward lymphoid-based responses. Furthermore, viral infections could lead to an increase in expression of the PD-1 ligand PD-L1 on cytotoxic T cells and other infected tissues, which, in the presence of blockade of PD-1 or PD-L1, may result in serious damage of healthy tissue by the immune system during COVID-19. All measures taken for immune-checkpoint blockade are supported only by indirect scientific literature and expert opinion and are therefore anecdotal. Most centers agree that in contrast to the strategy used for melanoma, immune-checkpoint blockade for patients with lung cancer should be continued (with stoppage rules to be discussed case by case, with the long half-life of immune-checkpoint blockades taken into consideration).

A further discussion point is whether or not to recommend prophylactic treatment with the cytokine G-CSF (granulocyte colony-stimulating factor) to patients at risk of neutropenia. Most centers recommend using it as primary prophylaxis for neutropenia-inducing therapies, especially during the COVID-19 pandemic, to boost the immune system. However, others worry that G-CSF stimulates the granulocytic response and may decrease the 


\section{Table 1 | General consensus measures taken by CCE centers during the COVID-19 pandemic}

\begin{tabular}{|c|c|}
\hline Category & Measure \\
\hline \multirow[t]{16}{*}{ Hospital wide } & Construct a hospital-wide crisis team responsible for coordinating measures between departments. \\
\hline & Encourage patients not to arrive early. Offer to text patients when you are ready to see them, so they can wait outside or in the car. \\
\hline & Instruct patients not to visit the hospital if they have symptoms indicative of possible COVID-19 (unless urgent attention is required). \\
\hline & Call patients the day before planned hospital admissions, to discuss the presence of any COVID-19-related symptoms. \\
\hline & Screen patients at the entrance for symptoms of COVID-19 and fever. \\
\hline & $\begin{array}{l}\text { Quickly isolate patients with COVID-19 in specialized departments, with the intent of relocation to regional collaborating hospitals } \\
\text { (if possible). }\end{array}$ \\
\hline & Reduce preclinical research activities to a bare minimum. \\
\hline & $\begin{array}{l}\text { Stop patient inclusion for clinical studies or trials requiring additional actions and/or visits. Consider a tumor type-specific 'exception } \\
\text { list' of particularly successful studies for which inclusion continues. }\end{array}$ \\
\hline & $\begin{array}{l}\text { Discuss each patient with a multidisciplinary team to consider alternative treatment modalities with the fewest visits or lowest } \\
\text { capacity problems or that are the shortest in duration. }\end{array}$ \\
\hline & Therapeutic adjustments (versus regular guidelines) should be discussed in a multidisciplinary team meeting. \\
\hline & $\begin{array}{l}\text { Conduct multidisciplinary team consultations remotely if possible or include only one representative of each discipline to limit the } \\
\text { number of people participating in the meetings. }\end{array}$ \\
\hline & Inform patients about a possibly increased risk associated with anticancer therapy during the COVID-19 pandemic. \\
\hline & Enable telephone or video consultations for healthcare professionals who need to self-isolate. \\
\hline & When postponing procedures or contact moments, anticipate future capacity problems. \\
\hline & $\begin{array}{l}\text { Do not prescribe corticosteroids as anti-emetics (if avoidable), and limit their use in patients treated with immune-checkpoint } \\
\text { blockade, to reduce vulnerability to COVID-19. }\end{array}$ \\
\hline & With each patient, discuss resuscitation status to anticipate future decisions about intensive care. \\
\hline \multirow[t]{6}{*}{ Outpatient clinic } & Critically triage second opinions. \\
\hline & Do all follow up appointments by phone (except when physical examination is necessary). \\
\hline & When possible, reduce or delay the number of radiological-response evaluations. \\
\hline & Prioritize oral or subcutaneous treatments above infusion-based treatments to reduce time spent in the hospital. \\
\hline & Perform blood tests outside the hospital (e.g., at a general practice or at home), when possible. \\
\hline & Have oral medications delivered to the patient's home, rather than being picked up at the pharmacy. \\
\hline \multirow[t]{3}{*}{ Day care } & Consider omitting supportive treatments (e.g., no bisphosphonate infusion, except in the case of hypercalcemia). \\
\hline & When possible, organize the administration of intravenous maintenance treatments at home. \\
\hline & $\begin{array}{l}\text { When administration at home is impossible, consider temporary breaks or reductions in the frequency of intravenous maintenance } \\
\text { treatments for less-aggressive metastatic cancers on a per-patient basis. }\end{array}$ \\
\hline \multirow[t]{2}{*}{ Radiotherapy } & Consider hypofractionated regimens for patients with limited additional benefit of regular regimens. \\
\hline & Create capacity for radiation as replacement of surgery. \\
\hline \multirow[t]{2}{*}{ Surgery } & Consider postponement of surgeries with high morbidity and mortality during the pandemic. \\
\hline & $\begin{array}{l}\text { Consider other treatment modalities with equal benefit (e.g., radiation for prostate cancer, curative chemoradiation for other tumor } \\
\text { types, or brain irradiation for metastases). }\end{array}$ \\
\hline Other & utsourcing of interventions (e.g., follow-up endoscopies) to private clinics. \\
\hline
\end{tabular}

lymphocytic response, whereas the latter may be mainly needed to fight COVID-19. Also, it has been reported that G-CSF levels were higher in patients with severe COVID-19 who required intensive care $^{12}$. Again, these conflicting opinions reflect the absence of solid scientific grounds for favoring one hypothesis over the other.

It is beyond the scope of this Perspective to describe all tumor-specific measures that have been adopted across the CCE centers, but we use breast cancer as an illustrative example. These recommendations are described in the accompanying supplementary information (Supplementary Table 1) and illustrate the various adaptations to the treatment of patients with breast cancer in our centers.

Patient information and psychosocial care. Patients with cancer generally understand the severity of the pandemic and its impact on healthcare systems in general and on their health in particular, so they are often willing to accept all suggested preventive measures. Anxiety among patients with cancer is currently high, nonetheless, and demand for counseling and mental-health assistance is skyrocketing. The most-frequent questions and concerns speak to the risk of patients becoming infected or suffering from severe complications of COVID-19 because of immunosuppression. In addition, patients are concerned that potential healthcare-capacity issues may interfere with the optimal treatment of their disease. Addressing patients' concerns poses another challenge to CCE centers, as it requires urgent attention in order to maintain high-quality cancer care. The national Cancer Information Service provided by the German Cancer Research Center, for example, has reported a substantial growth in patient inquiries related to their care during the pandemic. This demand was subsequently tackled by a combination of a short-term increase in personnel at the service, securing of 
Table 2 | The NHS scheme for prioritizing patients for systemic anticancer therapy by anticipated outcome

Priority level 1: Curative therapy with a high ( $>50 \%$ ) chance of success.

Adjuvant (or neo) therapy that adds at least $50 \%$ chance of cure to surgery or radiotherapy alone or treatment given at relapse.

Priority level 2: Curative therapy with an intermediate (20-50\%) chance of success.

Adjuvant (or neo) therapy that adds $20-50 \%$ chance of cure to surgery or radiotherapy alone or treatment given at relapse.

Priority level 3: Curative therapy of a low chance (10-20\%) of success Adjuvant (or neo) therapy that adds $10-20 \%$ chance of cure to surgery or radiotherapy alone or treatment given at relapse.

Non-curative therapy with a high ( $>50 \%$ ) chance of $>1$ year of life extension.

Priority level 4: Curative therapy with a very low (0-10\%) chance of success.

Adjuvant (or neo) therapy that adds a less than $10 \%$ chance of cure to surgery or radiotherapy alone or treatment given at relapse.

Non-curative therapy with an intermediate (15-50\%) chance of $>1$ year of life extension.

Priority level 5: Non-curative therapy with a high ( $>50 \%)$ chance of palliation/temporary tumor control but $<1$ year of life extension.

Priority level 6: Non-curative therapy with an intermediate (15-50\%) chance of palliation or temporary tumor control and $<1$ year of life extension.

more IT capacity, establishment of a professional and data protected online chat room in addition to the phone and email services - and by a joining of forces with the Infonet Cancer information system of German Cancer Aid. A regularly updated question-and-answer COVID-19 section was added to the website of the German Cancer Research Center Cancer Information Service, and this was communicated to the public by social-media campaigns and press releases. Other centers have provided similar support to patients.

Support of qualified personnel. For continuity of cancer care, the presence of sufficient qualified personnel to treat patients with cancer is essential. This involves the whole chain of hospital caregivers, from the operating theater to the ward, day clinic and intensive care unit (ICU). All CCE centers agreed that the absence of a rapid diagnostic system for assessing COVID-19 in caregivers is problematic. This frequently leads to (unnecessary) self-isolation of health professionals due to COVID-19-related symptoms, which further reduces the healthcare workforce in a time when demand is peaking. For the health system, the impact of an absence of 2-3 working days (the time currently needed for testing and self-quarantine) and a 6-hour absence (the time needed to be tested for COVID-19, if a quicker test were to be available) is enormous. With caregivers having a much greater chance of becoming infected and hence a substantial proportion of people unable to work, aggressive and quick testing of the healthcare workforce is a critical part of managing the crisis. Notably, reliance on these diagnostic tools could potentially result in a proportion of asymptomatic caregivers with 'false-negative' results returning to work and being capable of transmitting SARS-CoV-2 to patients and other caregivers - although only non-peer-reviewed evidence is currently available to support
Table 3 | The NHS scheme for prioritizing patients for surgery by anticipated outcome

Priority Emergency: operation needed within $24 \mathrm{~h}$ to save life level 1a: (e.g., perforated tumor)

Priority Urgent: operation needed within $72 \mathrm{~h}$ (e.g., obstruction not level 1b: able to be relieved by stent), based on:

Urgent/emergency surgery for life-threatening conditions such as obstruction, bleeding and regional and/or localized infection, or permanent injury/clinical harm from progression of conditions such as spinal cord compression

Priority Elective surgery with the expectation of cure prioritized level 2: according to:

within 4 weeks to save life/progression of disease beyond operability based on:

- urgency of symptoms

- complications such as local compressive symptoms - biological priority (expected growth rate) of individual cancers Local complications may be temporarily controlled, for example, with stents if surgery is deferred and/or interventional radiology

Priority Elective surgery can be delayed for 10-12 weeks with no level 3: predicted negative outcome

this hypothesis ${ }^{13}$. CCE centers would welcome and recommend widespread serological (immunoglobulin $G$ ) testing for past exposure (and presumed immunity), with return to work of previously infected caregivers a set number of days after the end of symptoms (if any), as a more-robust approach for the protection of patients and caregivers.

Capacity of cancer-care facilities. In many hospitals, the COVID-19 pandemic is a major stress test for the capacity of the various treatment or support units, including radiation, medical oncology, imaging and surgery units. Intensive-care capacity, including respiratory support, is the most challenged by COVID-19. A fraction of patients undergoing major surgery or systemic treatment for cancer require intensive-care support. Having COVID-19-infected patients in the ICU generally imposes an above-average burden, which reduces the capacity for non-COVID-19 patients in the ICU and hence a diminished ability to plan elective or emergency surgeries. This necessitates the discussion of alternative treatments such as radiation and/ or systemic therapies with patients with cancer.

All reviewed guidelines across the various CCE centers were unanimous in their recommendation that top priority must be given to neoadjuvant therapies and curative surgeries, when capacity on the operating room and/or ICU is limited. Alternatively, neoadjuvant systemic treatments may be initiated or extended to postpone surgical resections, or non-surgical interventions can be considered (e.g., definitive chemoradiation instead of resection for certain esophageal cancers, or neoadjuvant radiotherapy for breast-cancer management to delay or replace surgery). Also, several centers agree that certain elective surgeries can be delayed safely, when justified by scientific evidence; for example, an 11-week deferment of surgery for patients with rectal cancer after downstaging ${ }^{14}$ is deemed acceptable. However, when capacity remains sufficient, expediting elective surgical resection should be considered before a predicted surge in ICU bed occupancy - although such predictions can be challenging to make.

With increasing severity of the pandemic, healthcare systems will become overwhelmed, if they are not already, and prioritization will be necessary. To prepare for this, CCE centers have established 
Table 4 | The NHS scheme for prioritizing patients for radiotherapy by anticipated outcome

Priority Patients with category 1 (rapidly proliferating) tumors level 1: currently being treated with radical (chemo)radiotherapy with curative intent where there is little or no scope for compensation of gaps.

Patients with category 1 tumors in whom combined external beam radiotherapy (EBRT) and subsequent brachytherapy is the management plan and the EBRT is already underway.

Patients with category 1 tumors who have not yet started treatment and in whom clinical need determines that treatment should start in line with current cancer waiting times.

Priority Urgent palliative radiotherapy in patients with malignant level 2: spinal cord compression who have useful salvageable neurological function.

Priority Radical radiotherapy for category 2 (less-aggressive) tumors level 3: for which radiotherapy is the first definitive treatment.

Post-operative radiotherapy where there is known residual disease following surgery in tumors with aggressive biology.

Priority Palliative radiotherapy where alleviation of symptoms would level 4: reduce the burden on other healthcare services, such as hemoptysis.

Priority Adjuvant radiotherapy where there has been compete level 5: resection of disease and there is a $<20 \%$ risk of recurrence at 10 years; for example, most ER-positive breast cancer in patients receiving endocrine therapy.

Radical radiotherapy for prostate cancer in patients receiving neo-adjuvant hormone therapy.

decision rules to categorize and prioritize patients for systemic anticancer therapies or surgery. Although the exact details of such decision schemes differ by center, they are comparable and prioritize on the basis of anticipated treatment outcome. The most widely used guidance was that issued by the National Health Service (NHS) of England ${ }^{15}$ (systemic anticancer therapies, Table 2; cancer surgery, Table 3; radiotherapy, Table 4).

Research activities. The CCE centers have large research facilities and together employ thousands of preclinical scientists. One of the first measures taken was to downscale such preclinical research activities to a minimum in accordance with social-distancing guidelines and the local 'lockdown' policy. The priorities are to focus on maintaining infrastructure for core facilities (e.g., mouse facilities) and to avoid the disruption of lengthy experiments. Starting new experiments is generally discouraged. As for clinical research, the strategies have varied by center. Many centers halted the initiation of new clinical trials, as well as of studies and patient enrollment into clinical trials that require more resources than regular standard of care - such as additional interventions, imaging, contact moments or visits. Most scientists now work from home, and virtual meetings are used for the exchange of ideas and making plans for the future. Clinically trained scientists and research fellows are frequently going back to clinical work to support their healthcare system, a major bonus for centers struggling with, or anticipating, limitations of medical personnel. The current crisis will have major ramifications for the progress of cancer research. However, public-health measures in place to curtail the COVID-19 pandemic must be prioritized at present, and the damage to scientific enterprise will be repairable in time if safeguards and resources are put in place.

Organizational strategy to prepare for dynamic up- and downscaling. During the pandemic, cancer centers may need to swiftly
Table 5 | Phases used by the Netherlands Cancer Institute for downscaling scenarios during the COVID-19 pandemic

Phase Severity

Phase 0 $<15 \%$ disease rate of staff members

Phase 1 $15-30 \%$ disease rate of staff members

Phase 2 $30-50 \%$ disease rate of staff members

Phase 3 $>50 \%$ disease rate of staff members

and dynamically adapt their downscaling strategies. To this end, many centers have outlined scenarios to prepare for increasing (or decreasing) capacity problems using phased approaches. The Netherlands Cancer Institute, for example, has defined four phases of increasing severity (Table 5). For each phase, departments specified downscaling strategies based on the above-mentioned prioritization schemes. In general, centers established hospital-wide crisis teams to centrally coordinate their response to prevent conflicting department-specific strategies. Some countries have also established national coordination schemes. In Germany, capacity planning has been coordinated among 18 hospitals and the federal ministry of health, to prevent shortages of cancer services. We note that the optimal downscaling strategies depend on country- and center-specific capacities and preferences. Therefore, it is difficult to propose a common schedule, and it will be most effective if hospitals outline their own phase-specific downscaling strategies based on the prioritization schemes and practical handles discussed above.

\section{Preparing for the future}

Now, and probably even more so in the future, country-specific strategic choices for COVID-19 control are determining (and will determine) how and to what extent the oncological communities needs to re-organize their healthcare systems. As of 2 April 2020 (the time of this writing), countries have employed two fundamentally different approaches to control COVID-19: suppression (e.g., China, South Korea and, recently, Germany), versus mitigation (e.g., the Netherlands, UK, Spain, Sweden and Switzerland) ${ }^{16}$. This choice of strategy is critical for cancer centers, as it determines how profoundly oncological care should and will be reorganized during the pandemic. Although both strategies employ extensive transmission-reduction measures to control COVID-19, the fundamental difference is that suppression strategies aim to reach nearly complete suppression (and establish population immunity once a vaccine becomes available), whereas mitigation strategies aim to keep COVID-19 incidence consistently at the maximum levels acceptable in order to prevent overwhelming the healthcare systems - establishing population immunity with or without a vaccine.

From an oncological perspective, a suppression strategy would be preferred, as successful nearly complete suppression has the potential to make COVID-19-specific protocols for cancer care obsolete. However, so far, many countries have still preferred mitigation over suppression, and, with the current data, it is impossible to determine how long such mitigation will be needed to establish sufficient herd immunity. Specifically, as the number of asymptomatic people with COVID-19 is still a major unknown, the true incidence of COVID-19 is still unknown, and it remains unclear how quickly herd immunity to SARS-CoV-2 evolves. Nevertheless, the resolution of the current crisis may very well become a lengthy process, and oncologists urgently need models and data with which to enable systematic, evidence-based assessments of the risk/benefit ratio of anticancer therapies during the COVID-19 pandemic. For this goal to be achieved, two variables need to be known: (1) a treatment's added benefit for cancer control under normal conditions (ideally as an absolute risk reduction), and (2) a treatment's additional risk for COVID-19-related morbidity and mortality (ideally 
as an absolute risk increase). Although the first variable is known for many anticancer treatments, there are also many cases for which the effects of treatment modifications on cancer control are less clear, and it is critical that centers collect as much 'real-world' information as possible to quickly assess this. For the immediate future, however, the second variable represents the most pressing knowledge gap.

Quantifying COVID-19-related risks for patients with cancer. Estimating the increased risk associated with anticancer therapy during the COVID-19 pandemic again depends on two central variables: (1) a patient's risk of COVID-19 over the course of the anticancer therapy, and (2) a patient's additional risk for serious complications or death, should the patient become infected. At present, the uncertainty of the true incidence of COVID-19 (symptomatic cases plus asymptomatic cases) makes it impossible to accurately calculate a patient's risk of COVID-19 within a given timeframe (e.g., the length of the treatment). Determining the incidence of COVID-19 through the use of large-scale serological testing is therefore a priority. Once these data become available, country- or region-specific epidemiological models can be used to calculate the expected cumulative incidence of COVID-19 for patients with cancer as a function of time. Such models should factor in not only the local scale of the pandemic and mitigation factors such as ongoing lockdowns but also characteristics of the population of patients with cancer, such as age, frailty, mobility, co-morbidities and, perhaps, specific social-distancing measures.

We reason that the cumulative incidence for patients with cancer may be independent of potential immunosuppression by anticancer therapies, given that COVID-19-naive immunoproficient (healthy) people also lack effective immunity to SARS-CoV-2. In other words, it may be unlikely that anticancer therapies increase the incidence of COVID-19 in the population of patients with cancer, although data are needed to confirm this. However, anticancer treatments may increase the severity of COVID-19. In particular, chemotherapeutics that induce neutropenia and lymphopenia, targeted therapies that interfere directly or indirectly with the innate and adaptive immune system (e.g., tyrosine-kinase inhibitors, and inhibitors of the PIK-mTOR pathway) and immune-checkpoint inhibitors are postulated to worsen the course of COVID-19.

Critical research priorities. In order to better inform strategies to mitigate the impact of COVID-19 in the care of patients with cancer, we have identified the following four research priorities to enable evidence-based adjustment of anticancer regimens during the COVID-19 pandemic.

1. Collect real-world data on the effects of adjustment and de-escalation of treatment regimens on the outcomes of patients with cancer.

2. Determine the symptomatic and asymptomatic incidence of COVID-19 by large-scale serological testing in the general population and in patients with cancer who have been treated with chemotherapies, targeted therapies or immune-checkpoint inhibitors.

3. Develop an epidemiological model with which to estimate the cumulative incidence of COVID-19 for a patient with cancer within a specific timeframe.

4. Determine the COVID-19 morbidity and mortality in patients with cancer who are treated with chemotherapy, targeted therapy, immune-checkpoint blockade and/or G-CSF. To this end, several projects are underway, such as the UK Coronavirus Cancer Monitoring Project (https://ukcoronaviruscancermonitoring.com/).

The shadows ahead. This COVID-19 crisis has cast two long shadows on the future: first, there will be patients with less-favorable outcomes who will rightly demand an explanation for why they were treated in a different way, and second, the reprioritization of care will result in a queue of patients who need to undergo cancer treatment in rapidly growing waiting lists.

For healthcare professionals to be able to accurately inform patients about how the current treatment modifications impacted their outcomes, it is critical that as much real-world information as possible is collected. This may not only provide retrospective support for the adjustments that were made during the crisis but also provide valuable information on the effects of de-escalating regimens in general. In other words, the COVID-19 pandemic may offer a unique window of opportunity for retrospective trials, assessing the non-inferiority of de-escalated treatment regimens, which may be difficult to perform under normal conditions for ethical reasons. The oncological community should carefully think about how to use this opportunity and turn the risks that must be to taken today into benefits for patients in the future.

Dynamic up- and downscaling strategies with hospital-wide and national coordination may help to minimize potential future capacity problems by keeping productivity constantly at the maximum levels. However, this will probably be insufficient, and the community should already be starting to develop strategies to compensate for the current lower activity. This will be more challenging in countries in which healthcare systems are, under normal conditions, already used at very nearly their capacity.

\section{Conclusions}

By sharing our joint experiences, we have shown how the CCE centers aim to preserve continuity of cancer care amid the quickly evolving and multidimensional challenges posed by the COVID-19 pandemic. We have provided practical guidance for cancer centers and other hospitals to modify their current and dynamic operations in order to make them 'pandemic proof'. In brief, a hospital-wide crisis team could oversee and coordinate how each department implements the measures outlined in Table 1 and designs phase-specific prioritization blueprints using the schemes outlined in Tables 2-5. Furthermore, coordination with other regional or national hospitals may help in sharing the burden of care. Unfortunately, solid scientific data are often lacking to guide adjustments to standard-of-care treatment regimens. Whereas sharing and discussing the expert opinions of CCE centers may provide an initial roadmap for proceeding, the oncological community should quickly close key knowledge gaps about the incidence, morbidity and mortality of COVID-19 specific to patients with cancer, to enable evidence-based policies during this pandemic. In conclusion, we have provided a unique snapshot of a pressured re-organization of cancer healthcare systems at an unprecedented scale and pace, which we hope may offer guidance to other institutions and countries facing the same challenges.

Received: 4 April 2020; Accepted: 6 April 2020; Published online: 16 April 2020

\section{References}

1. Emanuel, E.J. et al. Fair allocation of scarce medical resources in the time of Covid-19. N. Engl. J. Med. (2020).

2. Calvo, F. et al. Cancer Core Europe: a European cancer research alliance realizing a research infrastructure with critical mass and programmatic approach to cure cancer in the 21st century. Eur. J. Cancer 103, 155-159 (2018)

3. Eggermont, A. M. et al. Cancer Core Europe: a consortium to address the cancer care-cancer research continuum challenge. Eur. J. Cancer 50, 2745-2746 (2014).

4. Eggermont, A. M. M. et al. Cancer Core Europe: a translational research infrastructure for a European mission on cancer. Mol. Oncol. 13, 521-527 (2019).

5. Coles, C. E. et al. International guidelines on radiation therapy for breast cancer during the COVID-19 pandemic. Clin. Oncol. 32, 279-281 (2020). 
6. Kunisaki, K. M. \& Janoff, E. N. Influenza in immunosuppressed populations: a review of infection frequency, morbidity, mortality, and vaccine responses. Lancet Infect. Dis. 9, 493-504 (2009).

7. Bitterman, R. et al. Influenza vaccines in immunosuppressed adults with cancer. Cochrane Database Syst. Rev. 2, CD008983 (2018).

8. Liang, W. et al. Cancer patients in SARS-CoV-2 infection: a nationwide analysis in China. Lancet Oncol. 21, 335-337 (2020).

9. Wang, H. \& Zhang, L. Risk of COVID-19 for patients with cancer. Lancet Oncol. 21, e181 (2020)

10. Xia, Y., Jin, R., Zhao, J., Li, W. \& Shen, H. Risk of COVID-19 for patients with cancer. Lancet Oncol. 21, e180 (2020).

11. Zhang, L. et al. Clinical characteristics of COVID-19-infected cancer patients: A retrospective case study in three hospitals within Wuhan, China. Ann. Oncol. https://doi.org/10.1016/j.annonc.2020.03.296 (2020).

12. Zhou, Y. et al. Pathogenic T cells and inflammatory monocytes incite inflammatory storm in severe COVID-19 patients. Natl. Sci. Rev. (2020)

13. Yang, Y. et al. Evaluating the accuracy of different respiratory specimens in the laboratory diagnosis and monitoring the viral shedding of 2019-nCoV infections. medRxiv https://doi.org/10.1101/2020.02.11.20021493 (2020).

14. Lefevre, J. H. et al. Effect of interval (7 or 11 weeks) Between neoadjuvant radiochemotherapy and surgery on complete pathologic response in rectal cancer: a multicenter, randomized, controlled trial (GRECCAR-6). J. Clin. Oncol. 34, 3773-3780 (2016).

15. National Health Service (NHS) England. Clinical guide for the management of non-coronavirus patients requiring acute treatment: Cancer (Publications approval reference: 001559). https://www.england.nhs.uk/coronavirus/ wp-content/uploads/sites/52/2020/03/specialty-guide-acute-treatment-cancer23-march-2020.pdf (2020).

16. Walker, P. et al. The global impact of COVID-19 and strategies for mitigation and suppression. Imperial College London https://doi.org/10.25561/77735 (2020).

\section{Acknowledgements}

We thank all members of CCE centers who contributed to the development of the guidelines presented in this perspective. A list of members of CCE centers is provided in the Supplementary Note. The Cancer Research UK Cambridge Center acknowledges funding from Cancer Research UK (Major Center Award A25117), the National Institute for Health Research Cambridge Biomedical Research Center, and The Mark Foundation for Cancer Research. Authors with the Netherlands Cancer Institute acknowledge the Dutch Cancer Society for funding. Authors with the Karolinska Institutet acknowledge Radiumhemmets Research Foundations, Stockholm, for funding. Authors with the Vall d'Hebron Institute of Oncology acknowledge the Cellex Foundation for providing research facilities and equipment and La Caixa Foundation and FERO Foundation for their funding support.

\section{Competing interests}

The authors declare no competing interests.

\section{Additional information}

Supplementary information is available for this paper at https://doi.org/10.1038/ s41591-020-0874-8.

Correspondence should be addressed to C.C. or E.E.V.

Reprints and permissions information is available at www.nature.com/reprints.

Publisher's note Springer Nature remains neutral with regard to jurisdictional claims in published maps and institutional affiliations.

Peer review Information Joao Monteiro was the primary editor on this article and managed its editorial process and peer review in collaboration with the rest of the editorial team.

(c) Springer Nature America, Inc. 2020 